PROCEEDINGS OF THE

AMERICAN MATHEMATICAL SOCIETY

Volume 137, Number 9, September 2009, Pages 3013-3024

S 0002-9939(09)09910-9

Article electronically published on May 4, 2009

\title{
ASYMPTOTICS FOR A GRADIENT SYSTEM WITH MEMORY TERM
}

\author{
ALEXANDRE CABOT \\ (Communicated by Walter Craig)
}

\begin{abstract}
Given a Hilbert space $H$ and a function $\Phi: H \rightarrow \mathbb{R}$ of class $\mathcal{C}^{1}$, we investigate the asymptotic behavior of the trajectories associated to the following dynamical system:

$$
\dot{x}(t)+\frac{1}{k(t)} \int_{t_{0}}^{t} h(s) \nabla \Phi(x(s)) d s=0, \quad t \geq t_{0},
$$

where $h, k:\left[t_{0},+\infty\right) \rightarrow \mathbb{R}_{+}^{*}$ are continuous maps. When $k(t) \sim \int_{t_{0}}^{t} h(s) d s$ as $t \rightarrow+\infty$, this equation can be interpreted as an averaged gradient system. We define a natural energy function $E$ associated to system $(\mathcal{S})$ and we give conditions which ensure that $E(t)$ decreases to inf $\Phi$ as $t \rightarrow+\infty$. When $\Phi$ is convex and has a set of non-isolated minima, we show that the trajectories of $(\mathcal{S})$ cannot converge if the average process does not "privilege" the recent past. Special attention is devoted to the particular case $h(t)=t^{\alpha}, k(t)=t^{\beta}$, which is fully treated.
\end{abstract}

\section{INTRODUCTION}

Given a Hilbert space $H$ with scalar product and corresponding norm respectively denoted by $\langle.,$.$\rangle and |$.$| , let us consider a smooth function \Phi: H \rightarrow \mathbb{R}$ that we wish to minimize. A powerful method consists in following the orbits of a continuous dynamical system, hopefully converging toward some minimizer of $\Phi$. The most classical one is the steepest descent equation

$$
\dot{x}(t)+\nabla \Phi(x(t))=0, \quad t \geq t_{0},
$$

which falls into the framework of the theory of dissipative dynamical systems (see, for example, [10, 11]). The trajectories of the above system are known to converge under various assumptions (Brézis-Bruck's theorem [5, 6], Lojasiewicz theorem [13]...)

The purpose of this paper is to analyse the effect of a memory term in the previous gradient system. More precisely, we study the following integro-differential equation:

$$
\dot{x}(t)+\frac{1}{k(t)} \int_{t_{0}}^{t} h(s) \nabla \Phi(x(s)) d s=0, \quad t \geq t_{0},
$$

Received by the editors October 22, 2008.

2000 Mathematics Subject Classification. Primary 34G20, 34A12, 34D05.

Key words and phrases. Differential equation, dissipative dynamical system, averaged gradient system, memory effect, Bessel equation.

(C)2009 American Mathematical Society Reverts to public domain 28 years from publication 
where $h, k:\left[t_{0},+\infty\right) \rightarrow \mathbb{R}_{+}^{*}$ are continuous maps. When $k(t) \sim \int_{t_{0}}^{t} h(s) d s$ as $t \rightarrow+\infty$, this equation can be interpreted as an averaged gradient system. In the recent papers [7, 8, special attention is devoted to the particular case corresponding to $h(t)=1, k(t)=t$ for $t \geq 0$, thus modelling a situation of uniform memory. When $\Phi$ is convex and has a set of non-isolated minima, it is proved in [7] that the nonstationary solutions cannot converge. This result is striking since the corresponding trajectories of the basic gradient system are convergent (at least weakly) under the same assumptions. We show in this paper that the trajectories of $(\mathcal{S})$ converge if and only if the weighted memory privileges enough the recent past. A precise quantification of this phenomenon is given in section 4 .

It is immediate that the solutions of $(\mathcal{S})$ satisfy the following second-order differential equation:

$$
k(t) \ddot{x}(t)+\dot{k}(t) \dot{x}(t)+h(t) \nabla \Phi(x(t))=0, \quad t \geq t_{0} .
$$

A key idea of the paper is that a suitable change of variable $t=\tau(s)$ allows us to rewrite equation (2) as follows:

$$
\ddot{y}(s)+a(s) \dot{y}(s)+\nabla \Phi(y(s))=0, \quad s \in\left[0, s_{\max }\right),
$$

where the map $y$ is defined by $y=x \circ \tau$. The quantity $s_{\max } \in \mathbb{R}_{+} \cup\{+\infty\}$ and the map $a:\left[0, s_{\max }\right) \rightarrow \mathbb{R}$ depend respectively on $h, k$ and their derivatives. Equation (3) has been recently studied in [7, 8, where the mechanical interpretation is emphasized via the use of a suitable energy function. When the map $a$ is constant, the underlying dynamical system is known under the terminology of "Heavy Ball with Friction" system. The (HBF) system has given rise to an abundant literature, see for example [2, 3, 12, and many results of convergence for the trajectories have been established under various assumptions such as convexity, analyticity,... .

In this paper, we exploit fully the recent results of [7, 8] to derive new results with respect to the generalized averaged gradient system $(\mathcal{S})$. Special attention is devoted to the particular case corresponding to $h(t)=t^{\alpha}, k(t)=t^{\beta}$, for $t \geq 1$. In this case, our general results allow us to determine the asymptotic behavior of $(\mathcal{S})$ for every couple $(\alpha, \beta) \in \mathbb{R}^{2}$. Moreover, when the corresponding equation is linear, it is shown in section 5 that its solutions can be explicitly computed via the Bessel functions.

\section{Global existence And Uniqueness}

Given a Hilbert space $H$ and a function $\Phi: H \rightarrow \mathbb{R}$ of class $\mathcal{C}^{1}$, let us consider the following dynamical system:

$$
\dot{x}(t)+\frac{1}{k(t)} \int_{t_{0}}^{t} h(s) \nabla \Phi(x(s)) d s=0, \quad t \geq t_{0},
$$

with initial data $x\left(t_{0}\right)=x_{0}$. If $h:\left[t_{0},+\infty\right) \rightarrow \mathbb{R}_{+}$and $k:\left[t_{0},+\infty\right) \rightarrow \mathbb{R}_{+}^{*}$ are continuous, then any solution $x$ of class $\mathcal{C}^{1}$ satifies $\dot{x}\left(t_{0}\right)=0$. Assuming moreover that $k$ is of class $\mathcal{C}^{1}$, let us multiply equation $(\mathcal{S})$ by $k(t)$ and let us differentiate; we obtain

$$
k(t) \ddot{x}(t)+\dot{k}(t) \dot{x}(t)+h(t) \nabla \Phi(x(t))=0 .
$$

If $\nabla \Phi$ is locally Lipschitz continuous, the Cauchy-Lipschitz theorem gives the local existence and uniqueness of a maximal solution $x$ of class $\mathcal{C}^{2}$ satisfying equation (4) 
and the initial conditions $\left(x\left(t_{0}\right), \dot{x}\left(t_{0}\right)\right)=\left(x_{0}, 0\right)$. The next statement summarizes the above analysis.

Proposition 2.1. Assume that $h:\left[t_{0},+\infty\right) \rightarrow \mathbb{R}_{+}$is continuous and that $k:$ $\left[t_{0},+\infty\right) \rightarrow \mathbb{R}_{+}^{*}$ is of class $\mathcal{C}^{1}$. Suppose also that the smooth function $\Phi: H \rightarrow \mathbb{R}$ is such that $\nabla \Phi$ is locally Lipschitz continuous. Given any $x_{0} \in H$, there exists a unique solution $x(.) \in \mathcal{C}^{2}\left(\left[t_{0}, T\right), H\right)$ of $(\mathcal{S})$ satisfying $x\left(t_{0}\right)=x_{0}$ on some maximal time interval $\left[t_{0}, T\right) \subset\left[t_{0},+\infty\right)$.

Given the change of variable $t=\tau(s)$, let us define the map $y$ by $y=x \circ \tau$. It is immediate to check that

$$
\dot{x}(t)=\frac{\dot{y}(s)}{\dot{\tau}(s)} \quad \text { and } \quad \ddot{x}(t)=\frac{\ddot{y}(s)}{\dot{\tau}(s)^{2}}-\dot{y}(s) \frac{\ddot{\tau}(s)}{\dot{\tau}(s)^{3}} .
$$

If we rewrite equation (4) by using the variable $s$ and the map $y$, we obtain after a division by $h(\tau(s))$,

$$
\frac{k(\tau(s))}{h(\tau(s))} \frac{\ddot{y}(s)}{\dot{\tau}(s)^{2}}+\frac{\dot{y}(s)}{h(\tau(s)) \dot{\tau}(s)}\left[\dot{k}(\tau(s))-k(\tau(s)) \frac{\ddot{\tau}(s))}{\dot{\tau}(s)^{2}}\right]+\nabla \Phi(y(s))=0 .
$$

Let us choose the map $\tau$ so we have $\dot{\tau}(s)=\sqrt{\frac{k(\tau(s))}{h(\tau(s))}}$. Let us define the map $F:\left[t_{0},+\infty\right) \rightarrow \mathbb{R}_{+}$by $F(t)=\int_{t_{0}}^{t} \sqrt{\frac{h(u)}{k(u)}} d u$ and let us set $s_{\max }=\int_{t_{0}}^{+\infty} \sqrt{\frac{h(u)}{k(u)}} d u \in$ $\mathbb{R}_{+} \cup\{+\infty\}$. The function $F:\left[t_{0},+\infty\right) \rightarrow\left[0, s_{\max }\right)$ defines an increasing one-toone map. It is then immediate that $\tau=F^{-1}$. From now on, let us assume that $h$, $k$ are both of class $\mathcal{C}^{1}$. By differentiating formula $\dot{\tau}(s)^{2}=\frac{k(\tau(s))}{h(\tau(s))}$, we easily obtain

$$
\ddot{\tau}(s)=\frac{\dot{k}(\tau(s)) h(\tau(s))-k(\tau(s)) \dot{h}(\tau(s))}{2 h(\tau(s))^{2}} .
$$

The differential equation (5) then becomes

$$
\ddot{y}(s)+a(s) \dot{y}(s)+\nabla \Phi(y(s))=0, \quad s \in\left[0, s_{\max }\right),
$$

where the map $a:\left[0, s_{\max }\right) \rightarrow \mathbb{R}$ is defined by

$$
a(s)=\frac{\dot{k}(\tau(s)) h(\tau(s))+k(\tau(s)) \dot{h}(\tau(s))}{2 h(\tau(s))^{\frac{3}{2}} k(\tau(s))^{\frac{1}{2}}} .
$$

By introducing the map $\eta:\left[t_{0},+\infty\right) \rightarrow \mathbb{R}$ defined by

$$
\eta=\frac{\dot{k} h+k \dot{h}}{h^{\frac{3}{2}} k^{\frac{1}{2}}}
$$

we then have $a=\frac{1}{2} \eta \circ \tau$. Notice that an alternative expression for the map $a$ is given by

$$
\begin{aligned}
a(s) & =\frac{\dot{k}(\tau(s)) h(\tau(s))+k(\tau(s)) \dot{h}(\tau(s))}{2 h(\tau(s)) k(\tau(s))} \dot{\tau}(s) \\
& =\frac{1}{2} \frac{d}{d s} \ln [h(\tau(s)) k(\tau(s))]
\end{aligned}
$$

The dynamical system (6) has been intensively studied in [7, 8]. Let us recall that the function $\mathcal{E}$ defined by $\mathcal{E}(s)=\frac{1}{2}|\dot{y}(s)|^{2}+\Phi(y(s))$ is a Lyapounov function associated to the trajectories of (6) . We define the corresponding energy function $E$ 
for the trajectories of $(\mathcal{S})$ by $E=\mathcal{E} \circ \tau^{-1}$, and its expression is given by $E(t)=$ $\frac{1}{2} \frac{k(t)}{h(t)}|\dot{x}(t)|^{2}+\Phi(x(t))$. The existence of the Lyapounov function $E$ is a crucial tool for deriving a result of global existence for the trajectories of $(\mathcal{S})$.

Proposition 2.2. Let $h, k:\left[t_{0},+\infty\right) \rightarrow \mathbb{R}_{+}^{*}$ be two maps of class $\mathcal{C}^{1}$ such that $h k$ is non-decreasing. Let $\Phi: H \rightarrow \mathbb{R}$ be a function of class $\mathcal{C}^{1}$ such that $\nabla \Phi$ is Lipschitz continuous on the bounded subsets of $H$. Assume moreover that the function $\Phi$ is bounded from below on $H$. Then, we have:

(a) There exists a unique maximal solution $x:\left[t_{0},+\infty\right) \rightarrow H$ of class $\mathcal{C}^{2}$ satisfying equation $(\mathcal{S})$ and the initial condition $x\left(t_{0}\right)=x_{0}$.

(b) For every $t \in\left[t_{0},+\infty\right)$, we have $\dot{E}(t)=-\frac{\dot{k}(t) h(t)+k(t) \dot{h}(t)}{2 h(t)^{2}}|\dot{x}(t)|^{2}$.

(c) If $\sqrt{\frac{h}{k}} \in L^{1}\left(\left[t_{0},+\infty\right)\right)$, then $|\dot{x}| \in L^{1}\left(\left[t_{0},+\infty\right)\right)$ and hence $\lim _{t \rightarrow+\infty} x(t)$ exists.

Proof. (a) Let $s_{\max }=\int_{t_{0}}^{+\infty} \sqrt{\frac{h(t)}{k(t)}} d t$. The assumptions on $h, k$ imply that the map $a:\left[0, s_{\max }\right) \rightarrow \mathbb{R}$ defined by (7) is non-negative and continuous. An immediate adaptation of [7, Prop. 2.2] shows that the maximal solution $y$ of the differential equation (6) satisfying $(y(0), \dot{y}(0))=\left(x_{0}, 0\right)$ is defined on $\left[0, s_{\max }\right)$. Hence the map $x:\left[t_{0},+\infty\right) \rightarrow H$ defined by $x=y \circ \tau^{-1}$ is the unique solution of class $\mathcal{C}^{2}$ satisfying equation $(\mathcal{S})$ and the initial condition $x\left(t_{0}\right)=x_{0}$.

(b) The announced formula follows by taking the scalar product of equation (4) against $\dot{x}(t) / h(t)$.

(c) The decay of the energy function $E$ combined with the fact that inf $\Phi>-\infty$ implies that

$$
\forall t \geq t_{0}, \quad \frac{k(t)}{h(t)}|\dot{x}(t)|^{2} \leq 2\left(E\left(t_{0}\right)-\inf \Phi\right) .
$$

Hence there exists $C \geq 0$ such that $|\dot{x}(t)| \leq C \sqrt{\frac{h(t)}{k(t)}}$. Since $\sqrt{\frac{h}{k}} \in L^{1}\left(\left[t_{0},+\infty\right)\right)$ by assumption, we conclude that $|\dot{x}| \in L^{1}\left(\left[t_{0},+\infty\right)\right)$.

Remark 2.1. When $\sqrt{\frac{h}{k}} \in L^{1}\left(\left[t_{0},+\infty\right)\right)$, the limit point $x_{\infty}=\lim _{t \rightarrow+\infty} x(t)$ is not in general a critical point of $\Phi$. The damping term $a(s)$ blows up as $s \rightarrow s_{\max }$, which forces the dynamics associated to (6) to stop at $x_{\infty}$ even if it is not a critical point of $\Phi$.

Example 2.1. Let us assume that $h(t)=t^{\alpha}$ and $k(t)=t^{\beta}$ for every $t \geq 1$. When $\alpha+\beta \geq 0$, the assumption that $h k$ is non-decreasing is satisfied and Proposition 2.2 applies. In particular, the result of existence and uniqueness holds. Condition $\sqrt{\frac{h}{k}} \in L^{1}([1,+\infty))$ is satisfied if and only if $\beta-\alpha>2$. In this case, we deduce from Proposition 2.2 (c) that $|\dot{x}| \in L^{1}([1,+\infty))$. The expression of the map $\eta$ defined by (8) is given by

$$
\forall t \geq 1, \quad \eta(t)=(\alpha+\beta) \frac{t^{\alpha+\beta-1}}{t^{\frac{3 \alpha}{2}} t^{\frac{\beta}{2}}}=(\alpha+\beta) t^{\frac{\beta-\alpha-2}{2}},
$$

for every $\alpha, \beta \in \mathbb{R}$. In particular, if $\beta-\alpha=2$ the map $\eta$ is constant and equal to $\alpha+\beta$. Since $a=\frac{1}{2} \eta \circ \tau$ we deduce in view of equality (6) that the map $y=x \circ \tau$ satisfies the following $(\mathrm{HBF})$ system:

$$
\ddot{y}(s)+\frac{\alpha+\beta}{2} \dot{y}(s)+\nabla \Phi(y(s))=0 .
$$


When $\beta-\alpha=2$, the solutions of system $(\mathcal{S})$ can then be viewed as re-parametrized $(\mathrm{HBF})$ trajectories, via the change of variable $t=\tau(s)$.

\section{Convergence of the EnERgy FunCtion}

The first result below provides a lower estimate for the quantity $E(t)-\inf \Phi$ as $t \rightarrow+\infty$. It is shown that if the map $h k$ does not tend toward $+\infty$, then the energy function cannot converge toward $\inf \Phi$ as $t \rightarrow+\infty$.

Proposition 3.1. Let $h, k:\left[t_{0},+\infty\right) \rightarrow \mathbb{R}_{+}^{*}$ be two maps of class $\mathcal{C}^{1}$ such that $h k$ is non-decreasing. Let $\Phi: H \rightarrow \mathbb{R}$ be a function of class $\mathcal{C}^{1}$ such that $\nabla \Phi$ is Lipschitz continuous on the bounded subsets of $H$. Assume moreover that the function $\Phi$ is bounded from below on $H$. Then, we have

$$
\forall t \geq t_{0}, \quad E(t)-\inf \Phi \geq\left(E\left(t_{0}\right)-\inf \Phi\right) \frac{h\left(t_{0}\right) k\left(t_{0}\right)}{h(t) k(t)} .
$$

If $\lim _{t \rightarrow+\infty} h(t) k(t)<+\infty$ and if $\Phi\left(x\left(t_{0}\right)\right)>\inf \Phi$, then $\lim _{t \rightarrow+\infty} E(t)>\inf \Phi$.

Proof. From [7, Prop. 2.6], the following inequality holds:

$$
\forall s \in\left[0, s_{\text {max }}\right), \quad \mathcal{E}(s)-\inf \Phi \geq(\mathcal{E}(0)-\inf \Phi) e^{-2 \int_{0}^{s} a(\sigma) d \sigma} .
$$

In view of expression (10), we have $\int_{0}^{s} a(\sigma) d \sigma=\frac{1}{2} \ln \frac{h(\tau(s)) k(\tau(s))}{h(\tau(0)) k(\tau(0))}$. Since $t=\tau(s)$ and $E=\mathcal{E} \circ \tau^{-1}$, inequality (13) follows directly. The last assertion is immediate.

The next result shows conversely that, if $\lim _{t \rightarrow+\infty} h(t) k(t)=+\infty$, then the energy function $E(t)$ satisfies a suitable property of summability, thus implying its convergence toward $\min \Phi$ as $t \rightarrow+\infty$.

Proposition 3.2. Let $h, k:\left[t_{0},+\infty\right) \rightarrow \mathbb{R}_{+}^{*}$ be two maps of class $\mathcal{C}^{2}$ such that $h k$ is non-decreasing and $\lim _{t \rightarrow+\infty} h(t) k(t)=+\infty$. Assume that the map $\eta=\frac{h \dot{k}+\dot{h} k}{h^{\frac{3}{2}} k^{\frac{1}{2}}}$ is non-increasing. Let $\Phi: H \rightarrow \mathbb{R}$ be a convex coerciv $€^{1}$ function of class $\mathcal{C}^{1}$ such that $\nabla \Phi$ is Lipschitz continuous on the bounded subsets of $H$. Then, the following estimate holds:

$$
\int_{t_{0}}^{+\infty} \frac{h(t) \dot{k}(t)+\dot{h}(t) k(t)}{h(t) k(t)}(E(t)-\min \Phi) d t<+\infty
$$

Moreover, we have $\lim _{t \rightarrow+\infty} E(t)=\min \Phi$. As a consequence, $\lim _{t \rightarrow+\infty} \frac{k(t)}{h(t)}|\dot{x}(t)|^{2}$ $=0$ and $\lim _{t \rightarrow+\infty} \Phi(x(t))=\min \Phi$.

Proof. Let us first prove that $s_{\max }=\int_{t_{0}}^{+\infty} \sqrt{\frac{h(t)}{k(t)}} d t=+\infty$. From the definition of the map $\eta$, we have

$$
\eta(t)=\frac{d}{d t} \ln (h(t) k(t)) \sqrt{\frac{k(t)}{h(t)}} .
$$

Since the map $\eta$ is non-increasing by assumption, we derive that

$$
\frac{d}{d t} \ln (h(t) k(t)) \leq \eta\left(t_{0}\right) \sqrt{\frac{h(t)}{k(t)}} .
$$

\footnotetext{
${ }^{1}$ Recall that the coercivity of $\Phi$ means that $\Phi(\xi) \rightarrow+\infty$ as $|\xi| \rightarrow+\infty$. This assumption combined with the convexity of $\Phi$ implies that $\arg \min \Phi \neq \emptyset$.
} 
Integrating the previous inequality on $\left[t_{0},+\infty\right)$ and recalling that $\lim _{t \rightarrow+\infty} h(t) k(t)$ $=+\infty$ by assumption, we conclude that $\int_{t_{0}}^{+\infty} \sqrt{\frac{h(t)}{k(t)}} d t=+\infty$.

The assumptions on $h, k$ imply that the map $a$ defined by (7) is non-negative, non-increasing and of class $\mathcal{C}^{1}$ on $\left[0, s_{\max }\right)=[0,+\infty)$. From [7, Prop. 3.1] we deduce that $\int_{0}^{+\infty} a(s)(\mathcal{E}(s)-\min \Phi) d s<+\infty$. It suffices then to replace $a(s)$ by its expression given by (9) and estimate (14) follows.

Let us now prove that $\lim _{t \rightarrow+\infty} E(t)=\min \Phi$. Let us argue by contradiction and assume that $\lim _{t \rightarrow \infty} E(t)>\min \Phi$. This implies the existence of $\varepsilon>0$ such that $E(t)-\min \Phi \geq \varepsilon$ for every $t \geq t_{0}$. We deduce that

$$
\begin{aligned}
\int_{t_{0}}^{+\infty} \frac{h(t) \dot{k}(t)+\dot{h}(t) k(t)}{h(t) k(t)}(E(t)-\min \Phi) d t & \geq \varepsilon \int_{t_{0}}^{+\infty} \frac{h(t) \dot{k}(t)+\dot{h}(t) k(t)}{h(t) k(t)} d t \\
& =\varepsilon[\ln (h(t) k(t))]_{t_{0}}^{+\infty}=+\infty,
\end{aligned}
$$

since $\lim _{t \rightarrow+\infty} h(t) k(t)=+\infty$. This contradicts estimate (14), which proves that $\lim _{t \rightarrow+\infty} E(t)=\min \Phi$. The last assertions are immediate.

Example 3.1. Take $h(t)=t^{\alpha}$ and $k(t)=t^{\beta}$ for every $t \geq 1$. Let us assume that $\alpha+\beta>0$ so that $\lim _{t \rightarrow+\infty} h(t) k(t)=+\infty$. Recalling formula (11), the map $\eta$ is non-increasing if and only if $\beta-\alpha \leq 2$. If both conditions $\alpha+\beta>0$ and $\beta-\alpha \leq 2$ hold and if the map $\Phi$ satisfies the same assumptions as in Proposition 3.2, we derive that $\lim _{t \rightarrow+\infty} E(t)=\min \Phi$.

In view of Proposition 3.2, we are able to investigate the question of the convergence of the trajectories in the case of a unique minimum. The case of non-isolated minima is more delicate and will be discussed in section 4.

Corollary 3.1. Under the hypotheses of Proposition [3.2, assume moreover that $\arg \min \Phi=\{\bar{x}\}$ for some $\bar{x} \in H$. Then any solution $x$ to the differential equation $(\mathcal{S})$ weakly converges to $\bar{x}$ in $H$.

The proof is classical and left to the reader. In the same direction, if we assume that $\bar{x}$ is a strong $y^{2}$ minimum for $\Phi$, we obtain a result of strong convergence to $\bar{x}$.

\section{The problem of CONVERGEnCE of trajectories For CONVEX POTENTIALS WITH NON-ISOLATED MINIMA}

We are going to investigate the question of convergence of the trajectories associated to $(\mathcal{S})$ when the potential $\Phi$ is convex and has non-isolated minima. Let us first consider the particular case $\Phi \equiv 0$. The differential equation (4) then becomes $k(t) \ddot{x}(t)+\dot{k}(t) \dot{x}(t)=0$ and a double integration immediately shows that its solution is given by $x(t)=x\left(t_{0}\right)+k\left(t_{0}\right) \dot{x}\left(t_{0}\right) \int_{t_{0}}^{t} \frac{d s}{k(s)}$. If $\dot{x}\left(t_{0}\right) \neq 0$, it ensues that the solution $x$ of equation (44) converges if and only if $\int_{t_{0}}^{+\infty} \frac{d s}{k(s)}<+\infty$. Therefore it is natural to ask whether for a general potential $\Phi$, the trajectory $x$ is convergent under this condition.

\footnotetext{
${ }^{2}$ We say that $\bar{x}$ is a strong minimum for $\Phi$ if for every $x \in H, \Phi(x) \geq \Phi(\bar{x})+\alpha(|x-\bar{x}|)$, where the map $\alpha: \mathbb{R}_{+} \rightarrow \mathbb{R}_{+}$is such that $\alpha\left(t_{n}\right) \rightarrow 0 \Longrightarrow t_{n} \rightarrow 0$ for every sequence $\left(t_{n}\right) \subset \mathbb{R}_{+}$.
} 
4.1. A general result of non-convergence. First, we give a general result of non-convergence for the trajectories of $(\mathcal{S})$ under the assumption $\int_{t_{0}}^{+\infty} \frac{d t}{k(t)}=+\infty$. This condition expresses that the map $k$ does not tend rapidly to infinity as $t \rightarrow$ $+\infty$. For example, when the map $k$ is of the form $k(t)=t^{\beta}$ for $t \geq 1$, the previous condition is satisfied if and only if $\beta \leq 1$. In the sequel, we assume that the convex function $\Phi$ satisfies the following geometrical condition at every $\bar{x} \in S=\arg \min \Phi$ : there exist a neighborhood $V$ of $\bar{x}$, a closed convex cone $K \subset H$ along with a positive real $\lambda>0$ such that

$\left(\mathcal{G C}_{\bar{x}}\right) \quad K \cap \mathbb{B} \subset \lambda(S-\bar{x}) \quad$ and $\quad-\nabla \Phi(x) \in K \quad$ for every $x \in V$,

where $\mathbb{B}$ denotes the closed unit ball of $H$. Recall that the normal cone $N_{S}(\bar{x})$ and the tangent cone $T_{S}(\bar{x})$ are respectively defined by

$$
N_{S}(\bar{x})=\{\xi \in H \mid \quad \forall x \in S, \quad\langle\xi, x-\bar{x}\rangle \leq 0\} ; \quad T_{S}(\bar{x})=\operatorname{cl}\left[\bigcup_{\lambda>0} \lambda(S-\bar{x})\right] .
$$

For further details relative to convex analysis, the reader is referred to Rockafellar's book [14. The geometric meaning of assertion $\left(\mathcal{G C}_{\bar{x}}\right)$ is brought to light by the following assertion: if $\operatorname{dim} H<+\infty$ and if

$$
-N_{S}(\bar{x}) \subset \operatorname{int}\left(T_{S}(\bar{x})\right) \cup\{0\}
$$

for some $\bar{x} \in S=\arg \min \Phi$, then condition $\left(\mathcal{G C}_{\bar{x}}\right)$ holds. For a proof of this result, the reader is referred to 9 . Condition (15) is satisfied in particular if the set $S$ is smooth 3 at $\bar{x} \in \operatorname{bd}(S)$. When $H=\mathbb{R}$, this condition is satisfied if and only if the interval $\arg \min \Phi$ is not a singleton.

Theorem 4.1. Let $h, k:\left[t_{0},+\infty\right) \rightarrow \mathbb{R}_{+}^{*}$ be two maps of class $\mathcal{C}^{1}$ such that $h k$ is non-decreasing. Assume moreover that

$$
\int_{t_{0}}^{+\infty} \frac{d t}{k(t)}=+\infty
$$

Let $\Phi: H \rightarrow \mathbb{R}$ be a convex function of class $\mathcal{C}^{1}$ such that $\nabla \Phi$ is Lipschitz continuous on the bounded sets of $H$. Assume that the geometric property $\left(\mathcal{G C}_{\bar{x}}\right)$ holds at every $\bar{x} \in S=\arg \min \Phi$. Given $x_{0} \in H$, consider the unique solution $x$ of $(\mathcal{S})$ satisfying $x\left(t_{0}\right)=x_{0}$. If $x_{0} \notin \arg \min \Phi$, then the trajectory $x$ of $(\mathcal{S})$ does not converge.

Proof. Let us first remark that the assumption $\int_{t_{0}}^{+\infty} \frac{d t}{k(t)}=+\infty$ implies that $s_{\max }=$ $\int_{t_{0}}^{+\infty} \sqrt{\frac{h(t)}{k(t)}} d t=+\infty$. Indeed, since the map $h k$ is non-decreasing, we have

$$
\int_{t_{0}}^{+\infty} \sqrt{\frac{h(t)}{k(t)}} d t=\int_{t_{0}}^{+\infty} \frac{\sqrt{h(t) k(t)}}{k(t)} d t \geq \sqrt{h\left(t_{0}\right) k\left(t_{0}\right)} \int_{t_{0}}^{+\infty} \frac{d t}{k(t)}=+\infty .
$$

We deduce from the assumptions on $h, k$ that the map $a$ defined by (7) is continuous and nonnegative on $\left[0, s_{\max }\right)=[0,+\infty)$. It suffices now to check that

\footnotetext{
${ }^{3}$ Recall that the set $S$ is smooth at $\bar{x} \in \operatorname{bd}(S)$ if there exists $d \neq 0$ such that $N_{S}(\bar{x})=\mathbb{R}_{+} d$.
} 
$\int_{0}^{+\infty} e^{-\int_{0}^{s} a(\sigma) d \sigma} d s=+\infty$ and then to apply [7, Theorem 4.1] 4 We have

$$
\begin{aligned}
\int_{0}^{+\infty} e^{-\int_{0}^{s} a(\sigma) d \sigma} d s & =\int_{0}^{+\infty} \sqrt{\frac{h\left(t_{0}\right) k\left(t_{0}\right)}{h(\tau(s)) k(\tau(s))}} d s \\
& =\sqrt{h\left(t_{0}\right) k\left(t_{0}\right)} \int_{0}^{+\infty} \frac{\dot{\tau}(s)}{k(\tau(s))} d s \\
& =\sqrt{h\left(t_{0}\right) k\left(t_{0}\right)} \int_{t_{0}}^{+\infty} \frac{d t}{k(t)}=+\infty
\end{aligned}
$$

which ends the proof.

Example 4.1. Let $\Phi: H \rightarrow \mathbb{R}$ be as in the previous theorem. Assume that $h(t)=t^{\alpha}$ and $k(t)=t^{\beta}$ for every $t \geq 1$. Theorem 4.1 shows that if $\alpha+\beta \geq 0$ and $\beta \leq 1$, then the non-stationary trajectories are divergent.

Remark 4.1. Coming back to the interpretation of $(\mathcal{S})$ as an averaged gradient system, let us assume that $k(t) \sim \int_{t_{0}}^{t} h(s) d s$ as $t \rightarrow+\infty$. In this case, condition (16) becomes

$$
\int_{t_{0}+1}^{+\infty} \frac{d t}{\int_{t_{0}}^{t} h(s) d s}=+\infty .
$$

For example, if $h(t)=t^{\alpha}(\ln t)^{\alpha^{\prime}}$ for every $t \geq 2$, condition (17) is satisfied if and only if $\alpha<0$ or $\left(\alpha, \alpha^{\prime}\right) \in\{0\} \times(-\infty, 1]$. Theorem 4.1 can be interpreted as follows: if the weighted memory associated to the density $h$ does not privilege enough the recent past, then the non-stationary trajectories of $(\mathcal{S})$ cannot converge. In particular, when the memory is uniform with respect to time (corresponding here to $\alpha=\alpha^{\prime}=0$ ), we recover the fact that non-stationary trajectories of $(\mathcal{S})$ are divergent.

Remark 4.2. Assume that $H=\mathbb{R}$, that $\arg \min \Phi=[c, d]$ with $c<d$, and that all other hypotheses of Theorem 4.1] hold. If $x_{0} \notin[c, d]$, the corresponding solution $x$ of $(\mathcal{S})$ does not converge and moreover the $\omega$-limit set $\omega\left(x_{0}\right)$ contains $[c, d]$. To see this, it suffices to apply the result of [7, Prop. 4.1].

4.2. A result of convergence in dimension one. We can now ask if the converse assertion is true: do the trajectories $x$ of $(\mathcal{S})$ converge under the condition $\int_{t_{0}}^{+\infty} \frac{d t}{k(t)}<+\infty$ ? In its full generality, this question is difficult and still open. We now give a positive answer in dimension one under a slightly stronger condition.

Theorem 4.2. Let $h, k:\left[t_{0},+\infty\right) \rightarrow \mathbb{R}_{+}^{*}$ be two maps of class $\mathcal{C}^{2}$ such that $h k$ is non-decreasing and tends to $+\infty$ as $t \rightarrow+\infty$. Assume that the map $\eta=\frac{h \dot{k}+\dot{h} k}{h^{\frac{3}{2}} k^{\frac{1}{2}}}$ is non-increasing and tends to 0 as $t \rightarrow+\infty$. Suppose moreover that there exists $\varepsilon>0$ such that

$$
\int_{t_{0}}^{+\infty} \frac{(h(t) k(t))^{\varepsilon}}{k(t)} d t<+\infty
$$

\footnotetext{
${ }^{4}$ In fact, the result of 7 . Theorem 4.1] is stated for a smooth set $S$, but its proof still works under the more general condition $(\mathcal{G C})$.
} 
Let $\Phi: \mathbb{R} \rightarrow \mathbb{R}$ be a convex function of class $\mathcal{C}^{1}$ such that $\Phi^{\prime}$ is Lipschitz continuous on the bounded sets of $\mathbb{R}$. Assume that $\arg \min \Phi=[c, d]$ with $c \leq d$ and that there exists $\delta>0$ such that

$$
\forall \xi \in(-\infty, c], \quad \Phi^{\prime}(\xi) \leq 2 \delta(\xi-c) \quad \text { and } \quad \forall \xi \in[d, \infty), \quad \Phi^{\prime}(\xi) \geq 2 \delta(\xi-d) .
$$

Then, any solution $x$ to the differential equation $(\mathcal{S})$ converges as $t \rightarrow+\infty$ toward some $x_{\infty} \in[c, d]$.

Proof. Let us first remark that the assumptions on $h, k$ imply that $s_{\max }=$ $\int_{t_{0}}^{+\infty} \sqrt{\frac{h(t)}{k(t)}} d t=+\infty$ (see the proof of Proposition 3.2 ). We also deduce from these assumptions that the map $a$ defined by (7) is of class $\mathcal{C}^{1}$ on $\left[0, s_{\max }\right)=[0,+\infty)$, it is non-negative, non-increasing and satisfies $\lim _{s \rightarrow+\infty} a(s)=0$. It suffices now to check that $\int_{0}^{+\infty} e^{-\theta \int_{0}^{s} a(\sigma) d \sigma} d s<+\infty$ for some $\theta<1$ and then to apply [8, Theorem 3.1]. Taking $\theta=1-2 \varepsilon$, we have

$$
\begin{aligned}
\int_{0}^{+\infty} e^{-\theta \int_{0}^{s} a(\sigma) d \sigma} d s & =\int_{0}^{+\infty}\left(\frac{h\left(t_{0}\right) k\left(t_{0}\right)}{h(\tau(s)) k(\tau(s))}\right)^{\frac{\theta}{2}} d s \\
& =\left(h\left(t_{0}\right) k\left(t_{0}\right)\right)^{\frac{1}{2}-\varepsilon} \int_{0}^{+\infty} \frac{(h(\tau(s)) k(\tau(s)))^{\varepsilon}}{k(\tau(s))} \dot{\tau}(s) d s \\
& =\left(h\left(t_{0}\right) k\left(t_{0}\right)\right)^{\frac{1}{2}-\varepsilon} \int_{t_{0}}^{+\infty} \frac{(h(t) k(t))^{\varepsilon}}{k(t)} d t<+\infty,
\end{aligned}
$$

which ends the proof.

Example 4.2. Let $\Phi: \mathbb{R} \rightarrow \mathbb{R}$ be as in the previous theorem. Assume that $h(t)=t^{\alpha}$ and $k(t)=t^{\beta}$ for every $t \geq 1$. Suppose that $\alpha+\beta>0$ and $\beta-\alpha<2$ so that we have $h(t) k(t) \nearrow+\infty$ and $\frac{h \dot{k}+h k}{h^{\frac{3}{2}} k^{\frac{1}{2}}} \searrow 0$ as $t \rightarrow+\infty$. On the other hand, if $\beta>1$, condition (18) is clearly satisfied with $\varepsilon=\frac{\beta-1}{2(\alpha+\beta)}$. Then we derive from the previous theorem that if $\beta>1$, any trajectory converges toward some minimum $x_{\infty}$ of $\Phi$.

Remark 4.3. Observe that in the previous example, the case $\beta-\alpha=2$ is not covered by Theorem 4.2. In this case, the map $y=x \circ \tau$ satisfies the (HBF) equation (12). If $\alpha+\beta>0$ and if $\Phi$ is convex with $\arg \min \Phi \neq \emptyset$, we know from [2, 3] that any trajectory $y$ of equation (12) weakly converges toward a minimum of $\Phi$. It ensues immediately that the result of Theorem 4.2 remains true when $\beta-\alpha=2$.

\section{Appendix: Study of a model example}

Throughout this section, we assume that $H=\mathbb{R}$ and we consider the linear differential equation

$$
t^{\beta} \ddot{x}(t)+\beta t^{\beta-1} \dot{x}(t)+t^{\alpha} x(t)=0, \quad t \geq 1,
$$

obtained from $(\mathcal{S})$ when $\Phi=\frac{1}{2}|\cdot|^{2}, h(t)=t^{\alpha}$ and $k(t)=t^{\beta}$ for every $t \geq 1$. By multiplying equation (19) by $t^{2-\beta}$ we obtain

$$
t^{2} \ddot{x}(t)+\beta t \dot{x}(t)+t^{\alpha-\beta+2} x(t)=0 .
$$

First assume that $\alpha-\beta+2=0$. In this case, we have $t^{2} \ddot{x}(t)+\beta t \dot{x}(t)+x(t)=0$, which is an Euler differential equation. After the change of variable $t=e^{s}$, the previous equation becomes a second-order ODE with constant coefficients. Setting 
$\Delta=\frac{(\alpha+\beta)^{2}}{4}-4$, we let the reader check that there exist $A, B \in \mathbb{R}$ such that for every $t \geq 1$,

$$
x(t)= \begin{cases}t^{-\frac{\alpha+\beta}{4}}\left[A \cos \left(\frac{\sqrt{-\Delta}}{2} \ln t\right)+B \sin \left(\frac{\sqrt{-\Delta}}{2} \ln t\right)\right] & \text { if }|\alpha+\beta|<4 \\ t^{-\frac{\alpha+\beta}{4}}[A+B \ln t] & \text { if }|\alpha+\beta|=4 \\ t^{-\frac{\alpha+\beta}{4}}\left[A t^{\frac{\sqrt{\Delta}}{2}}+B t^{-\frac{\sqrt{\Delta}}{2}}\right] & \text { if }|\alpha+\beta|>4 .\end{cases}
$$

Now assume that $\alpha-\beta+2 \neq 0$ and set $\mu=\frac{2}{\alpha-\beta+2}$. In this case, the change of variable $t=(s /|\mu|)^{\mu}$ shows that equation (20) is a transformed version of the Bessel differential equation. Standard references on Bessel equations are [1, 4]. We let the reader check that there exist $\hat{A}, \hat{B} \in \mathbb{R}$ such that for every $t \geq 1$,

$$
x(t)=t^{-\frac{\beta-1}{2}}\left[\hat{A} J_{\nu}\left(|\mu| t^{1 / \mu}\right)+\hat{B} Y_{\nu}\left(|\mu| t^{1 / \mu}\right)\right],
$$

where $\nu=\left|\frac{\beta-1}{\alpha-\beta+2}\right|$, and where $J_{\nu}, Y_{\nu}$ denote respectively the Bessel functions of the first and second kind. The coefficients $\hat{A}$ and $\hat{B}$ satisfy a linear system involving the initial data $\left(x_{1}, \dot{x}_{1}\right)=(x(1), \dot{x}(1))$ and by using the formula of Lommel 5 we easily obtain

$$
\begin{aligned}
& \hat{A}=\frac{\pi}{2}|\mu| x_{1} Y_{\nu}^{\prime}(|\mu|)-\frac{\pi}{2} \mu\left(\frac{\beta-1}{2} x_{1}+\dot{x}_{1}\right) Y_{\nu}(|\mu|), \\
& \hat{B}=\frac{\pi}{2} \mu\left(\frac{\beta-1}{2} x_{1}+\dot{x}_{1}\right) J_{\nu}(|\mu|)-\frac{\pi}{2}|\mu| x_{1} J_{\nu}^{\prime}(|\mu|) .
\end{aligned}
$$

Case $(\alpha-\beta+2>0)$. Then we have $\mu>0$; hence the variable $s=\mu t^{1 / \mu}$ tends to $+\infty$ as $t \rightarrow+\infty$. Recall that the asymptotic behavior of Bessel functions of the first and second kind is respectively given by

$$
J_{\nu}(s) \sim \sqrt{\frac{2}{\pi s}} \cos \left(s-\frac{\pi}{4}-\nu \frac{\pi}{2}\right) \quad \text { and } \quad Y_{\nu}(s) \sim \sqrt{\frac{2}{\pi s}} \sin \left(s-\frac{\pi}{4}-\nu \frac{\pi}{2}\right)
$$

as $s \rightarrow+\infty$. From the expression (21) for $x(t)$, we derive that

$$
x(t) \sim \hat{C} t^{-\frac{\beta-1}{2}-\frac{1}{2 \mu}} \cos \left(\mu t^{1 / \mu}-\hat{\phi}\right)=\hat{C} t^{-\frac{\alpha+\beta}{4}} \cos \left(\mu t^{\frac{\alpha-\beta+2}{2}}-\hat{\phi}\right),
$$

as $t \rightarrow+\infty$, for a suitable amplitude constant $\hat{C}$ and phase shift $\hat{\phi}$.

Case $(\alpha-\beta+2<0)$. Then we have $\mu<0$; hence the variable $s=|\mu| t^{1 / \mu}$ tends to 0 as $t \rightarrow+\infty$. Recall that the behavior of $J_{\nu}(s)$ and $Y_{\nu}(s)$ as $s \rightarrow 0$ is given by

$$
J_{\nu}(s) \sim \frac{1}{\Gamma(\nu+1)}\left(\frac{s}{2}\right)^{\nu} \quad \text { and } \quad Y_{\nu}(s) \sim \begin{cases}-\frac{\Gamma(\nu)}{\pi}\left(\frac{s}{2}\right)^{-\nu} & \text { if } \nu \neq 0 \\ \frac{2}{\pi} \ln s & \text { if } \nu=0\end{cases}
$$

where $\Gamma$ is the gamma function. From the expression (21) for $x(t)$, we derive that

- if $\beta>1$, then $\lim _{t \rightarrow+\infty} x(t)=-\hat{B} \frac{\Gamma(\nu)}{\pi}\left(\frac{|\mu|}{2}\right)^{-\nu}$,

- if $\beta=1$, then $x(t)=\left\{\begin{array}{cc}-\hat{B} \frac{2}{\pi|\mu|} \ln t+o(\ln t) & \text { if } \hat{B} \neq 0 \\ \hat{A}+o(1) & \text { if } \hat{B}=0\end{array}\right.$

\footnotetext{
${ }^{5}$ The formula of Lommel states that the Wronskian of the functions $J_{\nu}, Y_{\nu}$ is given by $J_{\nu}(s) Y_{\nu}^{\prime}(s)-J_{\nu}^{\prime}(s) Y_{\nu}(s)=\frac{2}{\pi s}$ for every $s>0$.
} 
- if $\beta<1$, then $x(t)=\left\{\begin{array}{cl}-\hat{B} \frac{\Gamma(\nu)}{\pi}\left(\frac{|\mu|}{2}\right)^{-\nu} t^{1-\beta}+o\left(t^{1-\beta}\right) & \text { if } \hat{B} \neq 0 \\ \hat{A}\left(\frac{|\mu|}{2}\right)^{\nu} \frac{1}{\Gamma(\nu+1)}+o(1) & \text { if } \hat{B}=0,\end{array}\right.$

as $t \rightarrow+\infty$. Formula (23) shows that the circumstance $\hat{B}=0$ is exceptional since it occurs only if $\left(\frac{\beta-1}{2} x_{1}+\dot{x}_{1}\right) J_{\nu}(|\mu|)+x_{1} J_{\nu}^{\prime}(|\mu|)=0$.

Let us now summarize all the possible asymptotic behaviors of the trajectory $x$, depending on $\alpha, \beta$ and the initial data. Denoting by $\bar{\omega}_{x_{1}, \dot{x}_{1}}$ the $\omega$-limit set as $t \rightarrow+\infty$ associated to the initial data $\left(x_{1}, \dot{x}_{1}\right) \in \mathbb{R}^{2} \backslash\{(0,0)\}$, we have

- If $\left\{\begin{array}{l}\alpha-\beta+2 \geq 0 \\ \alpha+\beta>0\end{array}\right.$ then $\lim _{t \rightarrow+\infty} x(t)=0$.

- If $\left\{\begin{array}{l}\alpha-\beta+2 \geq 0 \\ \alpha+\beta=0\end{array}\right.$ then $\bar{\omega}_{x_{1}, \dot{x}_{1}}=[-C, C]$, for some $C>0$.

- If $\left\{\begin{array}{l}\alpha-\beta+2>0 \\ \alpha+\beta<0\end{array}\right.$ or if $\left\{\begin{array}{l}\alpha-\beta+2=0 \\ \alpha+\beta \in(-4,0)\end{array}\right.$ then $\bar{\omega}_{x_{1}, \dot{x}_{1}}=(-\infty,+\infty)$.

- If $\left\{\begin{array}{l}\alpha-\beta+2=0 \\ \alpha+\beta \leq-4\end{array}\right.$ then $\lim _{t \rightarrow+\infty} x(t)= \pm \infty$.

- If $\left\{\begin{array}{l}\alpha-\beta+2<0 \\ \beta \leq 1\end{array}\right.$ then $\lim _{t \rightarrow+\infty} x(t)= \pm \infty$ or $\lim _{t \rightarrow+\infty} x(t)=l \in \mathbb{R}$.

- If $\left\{\begin{array}{l}\alpha-\beta+2<0 \\ \beta>1\end{array}\right.$ then $\lim _{t \rightarrow+\infty} x(t)=l^{\prime} \in \mathbb{R}$.

\section{ACKNOWLEDGEMENT}

The author would like to thank J. Droniou (University Montpellier II) for fruitful discussions and pertinent remarks about the paper.

\section{REFERENCES}

[1] M. Abramowitz, I. Stegun, Handbook of Mathematical Functions with Formulas, Graphs, and Mathematical Tables. Dover, New York, 1972. MR0167642 (29:4914)

[2] F. Alvarez, On the minimizing property of a second order dissipative system in Hilbert spaces, SIAM J. on Control and Optimization, 38 (2000), no. 4, 1102-1119. MR1760062 (2001e:34118)

[3] H. Attouch, X. Goudou, P. Redont, The heavy ball with friction method. I. The continuous dynamical system, Communications in Contemporary Mathematics, 2 (2000), no. 1, 1-34. MR:1753136 (2001b:37025)

[4] F. Bowman, Introduction to Bessel Functions. Dover, New York, 1958. MR0097539 (20:4007)

[5] H. Brézis, Opérateurs maximaux monotones et semi-groupes de contractions dans les espaces de Hilbert, Lecture Notes 5, North Holland, 1973. MR0348562 (50:1060)

[6] R.E. Bruck, Asymptotic convergence of nonlinear contraction semigroups in Hilbert space, Journal of Functional Analysis, 18 (1975), 15-26. MR0377609 (51:13780)

[7] A. Cabot, H. Engler, S. Gadat, On the long time behavior of second order differential equations with asymptotically small dissipation, accepted in Trans. of the Amer. Math. Soc. http://arxiv.org/abs/0710.1107

[8] A. Cabot, H. Engler, S. Gadat, Second order differential equations with asymptotically small dissipation and piecewise flat potentials, Seventh Mississippi State-UAB Conference on Differential Equations and Computational Simulations. Electronic Journal of Differential Equations, Conference 17 (2009), 33-38.

[9] A. Cabot, P. Frankel, Asymptotics for some proximal-like method involving inertia and memory aspects, 14 pages, submitted.

${ }^{6}$ As seen above, the second eventuality is exceptional and occurs only if $\hat{B}=0$. 
[10] J. K. Hale, Asymptotic behavior of dissipative systems. Mathematical Surveys and Monographs, 25. American Mathematical Society, Providence, RI, 1988. MR941371 (89g:58059)

[11] A. Haraux, Systèmes dynamiques dissipatifs et applications. RMA 17, Masson, Paris, 1991. MR:1084372 (92b:35002)

[12] A. Haraux, M. A. Jendoubi, Convergence of solutions of second-order gradient-like systems with analytic nonlinearities, J. Differential Equations, 144 (1998), no. 2, 313-320. MR1616968 (99a:35182)

[13] S. Lojasiewicz, Ensembles semi-analytiques réels, notes I.H.E.S., 1965.

[14] R.T. Rockafellar, Convex Analysis. Princeton Univ. Press, Princeton, NJ, 1970. MR 0274683 $(43: 445)$

Département de Mathématiques, Université Montpellier II, CC 051, Place Eugène Bataillon, 34095 Montpellier Cedex 5, France

E-mail address: acabot@math.univ-montp2.fr 